\title{
Radial Stiffness of a ferrofluid seal
}

\author{
R. Ravaud, M. Pinho, G. Lemarquand Senior IEEE, N. Dauchez, J.M. Genevaux, \\ V. Lemarquand and B. Brouard
}

\begin{abstract}
This paper presents an analytical model for calculating the shape and the radial stiffness of ferrofluid seals used as radial bearings and this theoretical value of the radial stiffness is compared to measured values. This approach is interesting for the design of loudspeakers. Moreover, the concept of magnetic pressure is used to determine the seal shape as well as its energy. This paper corresponds to the case in which the ferrofluid seal is submitted to a high magnetic field. Furthermore, the calculation of the seal shape when the moving part is decentred is discussed. Indeed, the variation of the seal energy is evaluated with a three-dimensional analytical approach and both the restoring force exerted by the seal and the radial stiffness are calculated. The results obtained with an experimental setting confirm the validity of the model.
\end{abstract}

Index Terms-ferrofluid seal, analytical calculation, magnetic energy, ironless loudspeaker

\section{INTRODUCTION}

$\mathbf{T}$ HE use of ferrofluids in loudspeakers is an interesting solution for the thermal dissipation as well as for the centering of the moving part [1][6]. Some authors have already studied the hydrostatical equilibrium of a ferrofluid in a magnetic field [7]-[10]. In this paper, the structure uses only neodymium iron boron permanent magnets that create high magnetic fields [11] which is not modified by the shape of the ferrofluid seal. Consequently, analytical calculations can be carried out to evaluate the magnetic field for all points in space [12][14]. Furthermore, the concept of magnetic pressure allows the determination of the seal shape as well as its energy for any position of the moving part.

Manuscript Received February 28, 2008. Corresponding author: G. Lemarquand (guy.lemarquand@ieee.org).

The authors are of the Laboratoire d'Acoustique de l'Universite du Maine UMR CNRS 6613, Avenue Olivier Messiaen, 72085 Le Mans Cedex 9, France
The seal shape is determined when the moving part is decentered and the calculation of the variation of the energy in the seal leads to the value of the restoring force exerted by the seal as well as its radial stiffness. Eventually, experiments are carried out to measure the force exerted by the seal when the moving part position changes radially. A good agreement is found between the theoretical model and the experimental setting.

\section{ShAPE AND RADIAL STIFFNESS OF THE FERROFLUID SEAL}

This section presents a three-dimensional model for studying the ferrofluid seal shape and its radial stiffness. This model is based on the analytical expressions of the axisymetric magnetic field components created by two ring permanent magnets.

\section{A. Expression of the magnetic pressure}

The shape and the radial stiffness of the ferrofluid seal can be studied by calculating the magnetic pressure $p_{m}(r, z)$ in the seal with $r$ and $z$ the radial and axial positions of the point. The ferrofluid is totally saturated and its magnetization is denoted $M_{s}$. Consequently, its magnetic relative permeability is 1 and the magnetic field produced by the magnetic particles is omitted. The ferromagnetic particles are assumed to be small saturated spheres that can be freely oriented in all the directions of space. Thus, all the particles of the saturated ferrofluid are aligned with the permanent magnet orienting field. For this study, the aggregation in chains of the ferrofluid particles is also omitted. Moreover, the thermal energy and the gravitational energy are neglected. 
The magnetic pressure in the ferrofluid seal is expressed as follows:

$$
\begin{aligned}
p_{m}(r, z) & =\mu_{0} \mathbf{M}_{\mathbf{s}} \mathbf{H}(r, z) \\
& =\mu_{0} M_{s} \sqrt{H_{r}(r, z)^{2}+H_{z}(r, z)^{2}}
\end{aligned}
$$

where $\mu_{0}$ is the permeability of the vacuum. The two magnetic field components $H_{r}(r, z)$ and $H_{z}(r, z)$ are analytically calculated and their threedimensional expressions are given in previous papers [15][16].

\section{B. Shape of the ferrofluid Seal}

The ferrofluid is contained between two cylindrical surfaces with two axial free boundary surfaces. Its shape depends mainly on the high magnetic field created by the ring permanent magnets. For the geometry chosen and the quantity of ferrofluid used, the magnetic iso-pressure surface near the free boundary surfaces are rather flat (Fig. 1). Thus, the contribution of the surface tension $\sigma=0.0256 \mathrm{~N} / \mathrm{m}$ can be neglected for the determination of the free surface. In this model, the seal free boundary surface is a magnetic iso-pressure surface. The ferrofluid seal shape is determined with its magnetic pressure $p_{m}(r, z)$. Figure 1 represents the isopressures of the ferrofluid. The regions where the magnetic pressure $p_{m}(r, z)$ is the most important are in front of the magnet junction at the right of the figure. When some ferrofluid is added to compose a seal, it first fills up the regions in space where the magnetic pressure is the most important. Consequently, the pressure at the free surface of the seal decreases when the ferrofluid volume increases.

\section{Obtaining the radial stiffness by using a three- dimensional model}

The radial stiffness of the ferrofluid seal can be obtained by calculating its magnetic potential energy in two configurations. The potential energies $E_{m}(0)$ and $E_{m}(e)$ are calculated when the nonmagnetic inner shaft is radially centered and when it is decentered of a value $e$ (Fig. 2). In the former configuration, the ferrofluid seal shape is given by

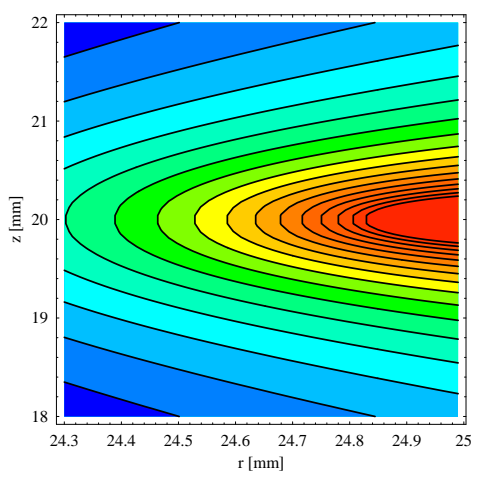

Fig. 1. Magnetic iso-pressure curves in the area of the seal, each ring permanent magnet height is $20 \mathrm{~mm}$. The regions where the magnetic pressure $p_{m}(r, z)$ is the most important are in front of the magnet junction at the right of the figure. The black lines define the seal contour, which depend on the ferrofluid volume. Indeed, the ferrofluid fills the areas at the right of the figure first (red ones). When the ferrofluid volume increases, the seal grows and occupies then the areas from the right to the left (yellow, green, and so on...).

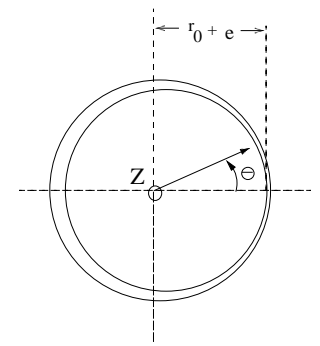

Fig. 2. Seal thickness for a decentered piston: $r_{i n}-r_{0}+$ $e \cos (\theta)$ where $r_{0}$ : piston radius, $r_{i n}$ : magnet internal radius, $e$ : decentering value. Seal nominal thickness: $r_{i n}-r_{0}$.

the surface of the decentered inner shaft and the free surfaces, where the axial position of the free surfaces remain the same and don't depend on $\theta$. Thus, the inner surface of the ferrofluid is given by

$$
r_{\text {out }}(\theta)=r_{0}+e \cos (\theta) .
$$

For a small gap, the axial limit $z_{0}$ can be approximated by a constant value. Thus, the difference of magnetic energy $\Delta E_{m}(e)-E_{m}(0)$ between the two configurations is given by the integrals:

$\Delta E_{m}=\int_{0}^{2 \pi} \int_{r_{0}+e \cos (\theta)}^{r_{i n}} \int_{-z_{0}}^{z_{0}} p_{m}(r, z) r d r d \theta d z$ 


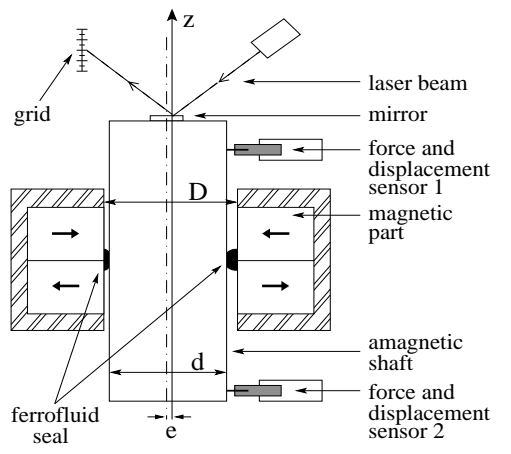

Fig. 3. Schematic diagram of the static stiffness measurement: crushing of the satured ferrofluid seals.

$$
-\int_{0}^{2 \pi} \int_{r_{0}}^{r_{i n}} \int_{-z_{0}}^{z_{0}} p_{m}(r, z) r d r d \theta d z
$$

With $r_{i n}$ the inner radius of the magnet. The work of the external radial force is equal to the variation of the energy. The force may not be proportional to the displacement $e$, but the radial stiffness can be evaluated by the first order development of this function :

$$
F_{r}=\frac{\Delta E_{m}}{2 e}
$$

This assumption is valid for small radial displacements of the inner piston.

\section{EXPERIMENTAL RESULTS}

An experimental study is performed to evaluate the radial stiffness of the ferrofluid seal located between a magnetic part and a non magnetic shaft. The experimental set-up is described in Fig. 3 and Fig. 4 shows a picture of the magnet assembly with ferrofluid seals.

The static part is a stack of two ring permanent magnets which are radially magnetized with opposite magnetization of $1.4 \mathrm{~T}$. The inner diameter is $D=49.70 \mathrm{~mm}$. The non magnetic shaft is a polymer rigid cylinder of diameter $d=49.00 \mathrm{~mm}$. In these conditions, the ferrofluid seal thickness is $0.35 \mathrm{~mm}$ at rest. Once the shaft and the ring magnets are centered, the ferrofluid

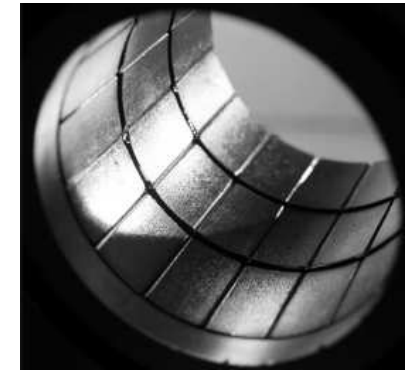

Fig. 4. Magnet assembly with two ferrofluid seals. In the experiment, only one seal is crushed by the moving part.

volume is added to form the seal. The experiments and the theoretical calculations are done for two different volumes of ferrofluid. This corresponds to two seal heights: $z_{0}=1 \mathrm{~mm}$ when the volume of ferrofluid is $V_{1}=105 \mathrm{~mm}^{3}$ and $z_{0}=1.7 \mathrm{~mm}$ for $V_{2}=188 \mathrm{~mm}^{3}$. The ferofluid tested is FerrotecAPGW05 $\left(\mu_{0} M_{s}=40 \mathrm{mT}\right)$. The theoretical values have been calculated for a displacement $e=0.1 \mathrm{~mm}$ (Table I).

The ferrofluid can be crushed in the radial direction by mean of two punctual forces $F_{1}$ and $F_{2}$ applied to the ends of the shaft. According to the choice of the forces, the movement of the shaft is composed by a radial displacement $e_{s}$, and an angular motion $\alpha$. To insure a pure translation displacement, a mirror is clamped on the upper shaft extremity and reflects a laser beam on a grid. The values of $F_{1}$ and $F_{2}$ are adjusted to obtain $\alpha=0$. The same displacement $e_{s}$ is imposed at each extremity of the shaft thank to the two displacement sensors mounted on micrometric positioning tables. The shaft is considered rigid, so this displacement is the same in the ferrofluid seal plane $e_{s}=e$. The force applied to the shaft, $F=F_{1}+F_{2}$, is measured with two specific non magnetic sensors using the strain gauge technique. The sensitivities of the sensors are $0.0026 \mathrm{~mm} / \mu \mathrm{def} \pm 0.01 \%$ for the displacement and $0.0019 \mathrm{~N} / \mu \mathrm{def} \pm 0.20 \%$ for the force. The ferrofluid static stiffness is determined by $k=F / e$.

The experimental uncertainties, $\triangle F$ and $\triangle e$, are obtained using the classical method of the propagation of errors formulas and account for the uncertainties of the sensor sensitivities and of 


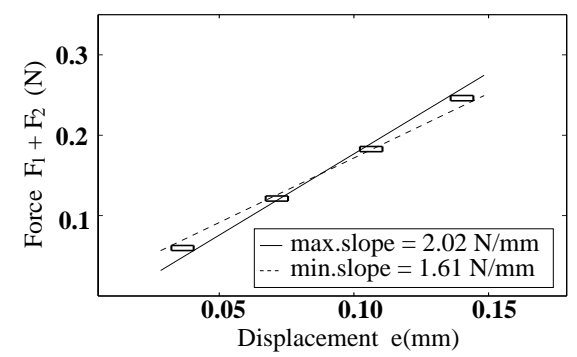

Fig. 5. Displacement of the non magnetic shaft versus the loading $\left(F_{1}+F_{2}\right)$ for Ferrotec-APGW05 and a volume $V_{1}=105.0 \mathrm{~mm}^{3}$

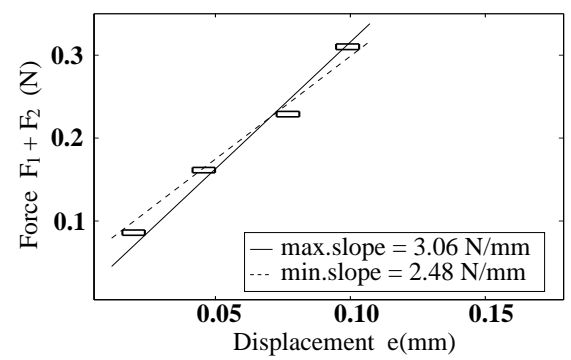

Fig. 6. Displacement of the non magnetic shaft versus the loading $\left(F_{1}+F_{2}\right)$ for Ferrotec-APGW05 and a volume $V_{2}=188.0 \mathrm{~mm}^{3}$

the driving displacement. These values are of the order of $\triangle F=0.0032 \mathrm{~N}$ and $\triangle e=0.0042 \mathrm{~mm}$ and give the size of the rectangle of uncertainty for each experimental point (Figs. 5,6). The overall uncertainty on $k$ is obtained from the maximum and minimum slope of the straight line that crosses all the rectangles. The measurement results are in good agreement with the predictions of the theoretical model (Tab. I).

\begin{tabular}{|c|c|c|c|}
\hline volume & $\mu_{0} M_{s}$ & $k_{\text {theo }}$ & $k_{\exp }$ \\
\hline$V_{1}=105 \mathrm{~mm}^{3}$ & $40 \mathrm{mT}$ & 1.85 & $1.82 \pm 0.21$ \\
\hline$V_{2}=188 \mathrm{~mm}^{3}$ & $40 \mathrm{mT}$ & 2.71 & $2.77 \pm 0.29$ \\
\hline
\end{tabular}

TABLE I

THEORETICAL AND EXPERIMENTAL STIFFNESSES OF THE FERROFLUID SEALS (IN $\mathrm{N} / \mathrm{mm}$ ) FOR THE DECENTERING VALUE $e=0.1 \mathrm{~mm}$

\section{CONCLUSION}

A three-dimensional analytical method is presented for calculating the shape and the radial stiffness of a ferrofluid seal. A good agreement is found between the theoretical model and the experimental measurements. Such an approach can be applied for the design of ironless structures using permanent magnets and ferrofluid.

\section{REFERENCES}

[1] R. E. Rosenzweig et al. "Study of audio speaker containing ferrofluid," J. Phys.: condens. matter, vol. 20, 2008.

[2] K. Raj et al. "Advances in ferrofluid in ferrofluid technology," JMMM, vol. 149, pp. 174-180, 1995.

[3] G. Lemarquand, "Ironless loudspeakers," IEEE Trans. Magn., vol. 43 (8), pp. 3371-3374, 2007.

[4] P. Kuzhir, "Free boundary of lubricant film in ferrofluid journal bearings," Tribology International, vol. 41, pp. 256268, 2008.

[5] O. Doutres et al. "On the use of a loudspeaker for measuring the viscoelastic properties of sound absorbing materials," JASA EL, vol. 124 (6), pp. 335-340, 2008.

[6] R. Ravaud et al. "Ironless loudspeakers with ferrofluid seals," Archives of Acoustics, vol. 33 (4), pp. 3-10, 2008.

[7] M. d'Aquino et al. "Forces in magnetic fluids subject to stationary magnetic fields," IEEE Trans. Magn., vol. 39 (5), pp. 2657-2659, 2003.

[8] R. C. Shah and M. Bhat, "Ferrofluid squeeze film in a long bearing," Tribology International, vol. 37, pp. 441446, 2004.

[9] S. H. Lee et al. "Indroducing the virtual air-gap scheme to the kelvin force densities with external and total field," IEEE Trans. Magn., vol. 43 (4), pp. 1457-1460, 2007.

[10] H. S. Choi et al."Simulation of hydrostatical equilibrium of ferrofluid subject to magneto-static field," IEEE Trans. Magn., vol. 44 (6), pp. 818-821, 2008.

[11] E. P. Furlani, Permanent Magnet and Electromechanical Devices. Academic Press, 2001.

[12] J. P. Selvaggi et al. "Computation of the three-dimensional magnetic field from solid permanent-magnet bipolar cylinders by employing toroidal harmonics," IEEE Trans. Magn., vol. 43 (10), pp. 3833-3839, 2007.

[13] S. Babic et al."Calculation improvement of 3d linear magnetostatic field based on fictitious magnetic surface charge," IEEE Trans. Magn., vol. 36 (5), pp. 3125-3127, 2000.

[14] R. Ravaud et al."Discussion about the analytical calculation of the magnetic field created by permanent magnets.," PIER $B$, vol. 11, pp. 281-297, 2009.

[15] R. Ravaud et al."Analytical calculation of the magnetic field created by permanent-magnet rings," IEEE Trans. Magn., vol. 44 (8), pp. 1982-1989, 2008.

[16] R. Ravaud et al."The three exact components of the magnetic field created by a radially magnetized tile permanent magnet.," PIER, vol. 88, pp. 307-319, 2008. 